\title{
DESENVOLVIMENTO DE PROGRAMAS NO SOFTWARE EMSO PARA A MODELAGEM E A DISCRIMINAÇÃO DE MODELOS CINÉTICOS APLICADOS À SECAGEM DE MILHO
}

\author{
G.M. PETRUCELLI ${ }^{1}$ e M.C. MANCINI ${ }^{1}$ \\ ${ }^{1}$ Universidade Federal Rural do Rio de Janeiro, Departamento de Engenharia Química \\ e-mail para contato: mancinimc@gmail.com; petrugeral@gmail.com
}

\begin{abstract}
RESUMO - O presente trabalho objetivou a implementação de rotinas para simulação e estimativa de parâmetros, utilizando o software EMSO, com vistas ao desenvolvimento de uma metodologia didática para facilitar o estudo da cinética de secagem de grãos de milho em camada delgada. Foram avaliados os modelos de Overhults, bem como, os modelos de taxas de secagem de primeiro e de segundo graus, para descrever este tipo de operação. Os dados experimentais utilizados neste trabalho foram obtidos de trabalhos disponíveis na literatura, uma vez que o foco da pesquisa é a utilização do software EMSO, como uma ferramenta didática para o estudo da secagem de grãos. A partir dos resultados obtidos com a estimativa de parâmetros, foi possível comparar os resultados experimentais com os resultados simulados, obtidos por meio de cada um dos modelos. Por fim, foi feita a discriminação do modelo cinético que melhor descreve o processo, na faixa de condições experimentais estudadas, com base nos critérios de minimização do desvio relativo médio e dos maiores valores do coeficiente de determinação $\left(\mathrm{R}^{2}\right)$. Verificou-se que modelo de Overhults foi o mais adequado para descrever a cinética de secagem de milho em camada delgada, ou seja, apresentou os menores desvios relativos médios (DRM < 1,56) e os maiores valores de coeficiente de determinação $\left(\mathrm{R}^{2}>0,99\right)$, para todos os experimentos considerados neste estudo.
\end{abstract}

\section{INTRODUÇÃO}

\subsection{Secagem}

A secagem ou desidratação é o método mais antigo empregado em conservação de alimentos e tem por objetivo principal eliminar, por evaporação, a maior parte da água presente no produto. A redução do teor de umidade inibe ou reduz a ação microbiana e a atividade enzimática do alimento, que, de outra forma, poderia causar danos ao mesmo. A desidratação, também, torna o manuseio do produto alimentício mais fácil, devido à contração de volume e diminuição da massa, reduzindo as perdas durante o transporte (Ochoa et al., 2002).

Entretanto, a remoção de água, conduzida a altas temperaturas e longos períodos de secagem, pode causar a perda do valor nutritivo e sensorial, desnaturação de nutrientes, escurecimento do material e mudanças na consistência e no sabor do alimento, exigindo 
estudos e pesquisas mais extensivos e detalhados para uma melhor compreensão do processo (Mandala et al., 2005).

\subsection{O software EMSO}

A ferramenta utilizada, o software EMSO (Enviroment for Modeling, Simulation and Optimization), é uma plataforma desenvolvida para modelar, simular e otimizar preferencialmente processos em sistemas dinâmicos, podendo ser facilmente adaptada para a descrição de processos em sistemas estáticos ou em regime permanente. É possível a criação de três tipos de estruturas de programação para a descrição do processo alvo: "models", que seriam a descrição matemática do dispositivo ("device") utilizado no processo; "devices", que seriam os instrumentos utilizados no processo, como tanques e/ou reatores; e, por fim, o "flowsheet", que seria o fluxograma do processo, representando o conjunto de dispositivos com os modelos anexados neles. (Soares, 2007)

A estimativa de parâmetros dos modelos introduzidos ao programa pode ser feita por meio do comando "Estimation". Este comando realiza a estimativa com base nos dados experimentais de um processo, usando o método dos mínimos quadrados já introduzidos na plataforma. É necessário apenas estabelecer uma faixa de busca, sendo mais estreita para modelos cinéticos com muitos mínimos e máximos locais; e mais larga para modelos com mínimos e máximos locais espaçados. Após o fim de tal processo, o programa fornece valores de $\mathrm{R}^{2}$ para o modelo e quantifica a significância dos parâmetros. (Soares, 2007)

Com o auxílio das utilidades oferecidas pelo EMSO, é possível obter métodos de comparação de maneira simples, mais rápidos e mais precisos que os meios convencionais. Mecanismos como a geração de gráficos, análises estatísticas e a forma simples e clara de explicitar dados, são princípios muito úteis para tal finalidade didática deste projeto. Assim, esta ferramenta possibilita uma avaliação apurada da capacidade dos modelos em descrever adequadamente o processo utilizado.

Com amplos recursos de modelagem e simulação de simples acesso, o EMSO se mostra uma ferramenta didática poderosa. Uma vez que processos, tanto dinâmicos quanto estáticos, podem ser implementados facilmente em um programa, problemas didáticos se tornam de mais fácil entendimento.

\section{MATERIAL E MÉTODOS}

\subsection{Modelos Matemáticos}

Para a criação dos programas simuladores, foram estudados três modelos matemáticos: Modelo empírico de Overhults (Overhults et al., 1973), Equação 1, modelo de taxa de transferência de massa de $1^{\circ}$ Grau, que corresponde ao balanço de massa de água no grão, considerando a constante de secagem $(\mathrm{k})$ independente das condições de operação e da umidade do material, Equação 2; e modelo de taxa de transferência de massa de $2^{\circ}$ Grau, em que a constante k é considerada uma função linear da umidade do material, Equação 3.

$$
\begin{aligned}
& Y s(t)=Y s e+(Y s o-Y s e) e^{-(k t)^{n}} \\
& \frac{d Y s(t)}{d t}=-k(Y s(t)-Y s e)
\end{aligned}
$$




$$
\frac{d Y s(t)}{d t}=-(A . Y s(t)-B)(Y s(t)-Y s e)
$$

Onde: Ys(t) é a umidade do material no instante t; Yse é a umidade de equilíbrio do material; Yso é a umidade inicial do material e k, n, A e B são parâmetros referentes a cada modelo.

Para descrever a umidade de equilíbrio, foi utilizada a Equação 4 obtida na literatura (MANCINI, 1996).

$$
Y s e=\left[\frac{-\ln (1,0-U R)}{120,6(T s+45,6)}\right]^{0,5}
$$

Onde: Ts é a temperatura de secagem em ${ }^{\circ} \mathrm{C}$ e UR é a umidade relativa do gás de secagem.

\subsection{Criação do "flowsheet" simulador}

Após estudar os modelos cinéticos expostos acima, o software EMSO, versão beta 0.10.6, para plataforma Windows 10, 64 bits, foi utilizado para a implementação de "flowsheets" para cada modelo, apresentando suas equações com seus respectivos parâmetros, variáveis e condições iniciais e de operação. Em cada simulação se estabelece, também, o tempo inicial e final, bem como o tamanho do intervalo de coleta de dados. Um fragmento de programa, descrevendo a simulação da secagem em camada delgada, com cinética descrita pelo modelo de Overhults, é apresentado na Figura 1.a. Nos casos dos outros dois modelos avaliados, apenas a equação descritiva do modelo foi modificada para o caso específico.

Figura 1 - Fragmentos de programa do EMSO descrevendo: (a) a rotina de simulação da

\begin{tabular}{|c|c|}
\hline end & $\begin{array}{l}\text { FlowSheet Secagem_Milho_Overhults } \\
\text { PARAMETERS } \\
\text { UR as Real (Brief="Umidade relativa do ar"); } \\
\text { k as Real (Brief="Parâmetro k - cte de secagem"); } \\
\text { n as Real (Brief="Parâmetro n"); } \\
\text { Ts as Real (Brief="Temperatura",Unit='K'); } \\
\text { Yso as Real (Brief="umidade inicial"); } \\
\text { VARIABLES } \\
\text { Ys as Real (Brief="Umidade de base seca no instante t"); } \\
\text { Yse as Real (Brief="Umidade de equilíbrio"); } \\
\text { SET } \\
\text { \# Condições de Operação } \\
\text { Ts = 48.7*'K'; } \\
\text { UR = 0.21; } \\
\text { Yso = 0.2144; } \\
\text { k=0.00353312; } \\
\text { n= 0.524259; } \\
\text { SPECIFY } \\
\text { \# "Umidade de equilibrio" } \\
\text { Yse = (-ln(1-UR)/120.6/(Ts/'K'+45.6))^(0.5); } \\
\text { EQUATIONS } \\
\text { "MODELO DE OVERHULTS" } \\
\text { Ys = Yse + (Yso - Yse)*exp(-((k*(time *'1/min'))^n)); } \\
\text { OPTIONS } \\
\text { TimeStep = 5; } \\
\text { TimeEnd = 180; } \\
\text { TimeUnit = 'min'; }\end{array}$ \\
\hline
\end{tabular}
secagem, (b) a rotina de estimativa de parâmetros, ambas usando o modelo de Overhults.

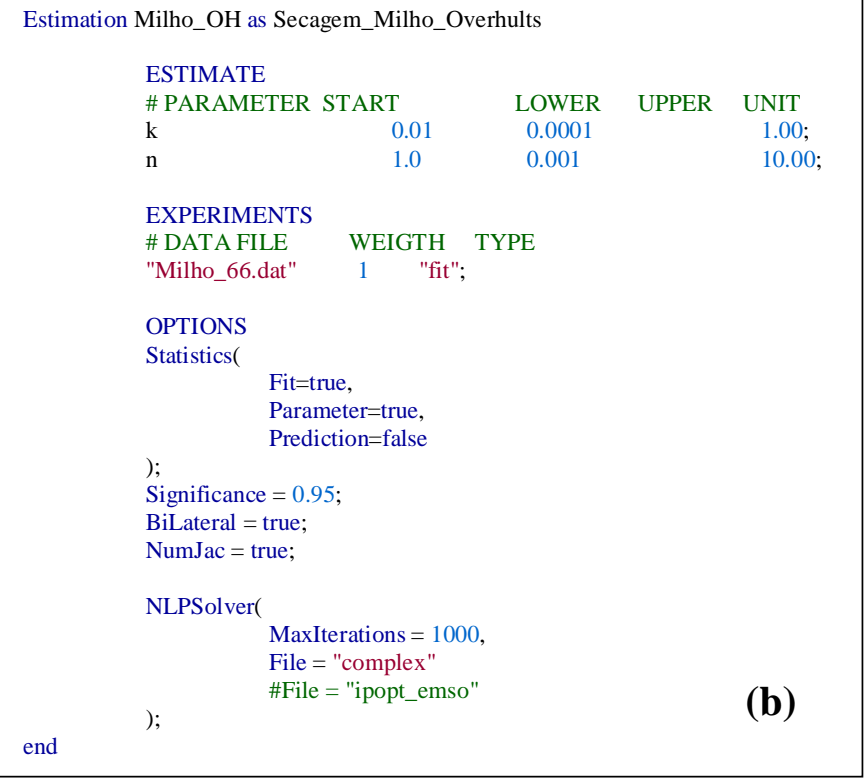


O desenvolvimento das rotinas de simulação consistiu apenas da primeira etapa deste projeto. Uma vez que foi possível simular a secagem de grãos usando um dos três modelos estudados, de forma simples, com interface amistosa com o usuário, foi necessário ampliar o uso da ferramenta didática, inserindo as rotinas de estimativa de parâmetros, para que o estudo pudesse confrontar resultados simulados e experimentais, possibilitando ao usuário efetuar a discriminação do modelo mais adequado para descrever o processo.

\subsection{Estimativa dos parâmetros}

Para a estimativa dos parâmetros, os resultados experimentais de secagem, obtidos do trabalho de Mancini (1996) foram tabulados em arquivos de dados formatados segundo as exigências da ferramenta "Estimation" do EMSO.

A estimativa dos parâmetros no EMSO é baseada na utilização de uma "flowsheet" operacional para a simulação do processo, como desenvolvido na etapa anterior deste projeto. A partir da "flowhseet" simuladora funcional, a ferramenta "Estimation" pode ser facilmente implementada, conforme pode ser observado no fragmento de programa apresentado na Figura 1.b.

Para os modelos utilizados, foram necessárias duas faixas de busca nos modelos de Overhults e $2^{\underline{o}}$ Grau, uma vez que possuem dois parâmetros a serem estimados em sua descrição matemática; e uma faixa para o modelo de $1^{\circ}$ Grau, possuindo apenas um parâmetro a ser estimado.

Após a estimativa de parâmetros, o software fornece o coeficiente de determinação $\left(\mathrm{R}^{2}\right)$, o indicador de significância de cada parâmetro e os resultados preditos da variável dependente.

\subsection{Análise de dados e discriminação de modelos}

Após a implementação das rotinas de simulação e estimativa de parâmetros, os programas do EMSO foram avaliados, para o caso da secagem de milho em camada delgada, com vistas à discriminação do modelo matemático que melhor descreve o processo.

Os dados referentes a 70 experimentos de secagem de milho em camada delgada foram obtidos no trabalho de Mancini (1996), no qual o autor realizou um abrangente estudo experimental da secagem de grãos de milho, soja e feijão em camada delgada e de milho em soja em camada espessa, determinando as curvas de cinética de secagem dos referidos grãos em variadas condições de operação. As faixas de condições operacionais da secagem de milho em camada delgada são apresentadas na Tabela 1.

Tabela 1 - Condições de operação na secagem de milho em camada delgada (Mancini, 1996)

\begin{tabular}{|c|c|}
\hline Variável & Faixas \\
\hline Temperatura $\left({ }^{\circ} \mathrm{C}\right)$ & $29,7 \leq \mathrm{T} \leq 70,1$ \\
\hline Umidade Relativa (UR) & $0,06 \leq \mathrm{UR} \leq 0,67$ \\
\hline Umidade inicial (Yso) & $0,1911 \leq$ Yso $\leq 0,3979$ \\
\hline Tempo de Secagem (min) & 180 \\
\hline
\end{tabular}




\section{RESULTADOS E DISCUSSÃO}

A partir da simulação realizada pelo programa, com os parâmetros estimados, foram obtidos os desvios relativos médios e coeficientes de determinação para cada modelo utilizando os resultados das 70 curvas de secagem apresentadas no trabalho de Mancini (1996). O modelo cinético de Overhults apresentou os menores desvios, variando de 0,08 até $1,56 \%$, e maiores coeficientes de determinação, todos acima de 0,99 . O modelo de $2^{\circ}$ Grau também apresentou resultados satisfatórios, com desvios entre 0,69 e 4,94\%; todos inferiores às incertezas experimentais, que foram de aproximadamente $5 \%$; além de obter valores de $\mathrm{R}^{2}$ maiores que 0,97 . Já o modelo de $1^{\circ}$ Grau apresentou coeficientes de determinação a partir de 0,94 e desvios entre 3,75 e $17,28 \%$, não se apresentando como um modelo indicado para simular este tipo de processo. Em todos os casos e modelos estudados, os parâmetros sempre foram significativos.

Nas Figuras 2.a e 2.b são apresentados os resultados das comparações entre valores observados e preditos com cada um dos modelos estudados, referentes à melhor e à pior situação encontrada no conjunto de dados experimentais disponíveis. Em todos os casos, verificou-se, por meio de análise variância e testes de médias, com $\mathrm{p}<0,05$, ou seja, $95 \%$ de confiança, que as diferenças observadas e os parâmetros determinados são significativos.

Figura 2 - Comparação entre resultados simulados e observados da umidade do milho. (a) Resultados referentes à simulação do experimento que apresentou menores desvios relativos médios e maiores valores de $\mathrm{R}^{2}$ ( $\mathrm{UR}=0,44 ; \mathrm{T}=35^{\circ} \mathrm{C}$ e Yso $\left.=0,2498\right)$. (b) Resultados referentes à simulação do experimento que apresentou os maiores desvios relativos médios $\mathrm{e}$ menores valores de $\mathrm{R}^{2}\left(\mathrm{UR}=0,07 ; \mathrm{T}=70,1{ }^{\circ} \mathrm{C}\right.$ e $\left.\mathrm{Yso}=0,2622\right)$.
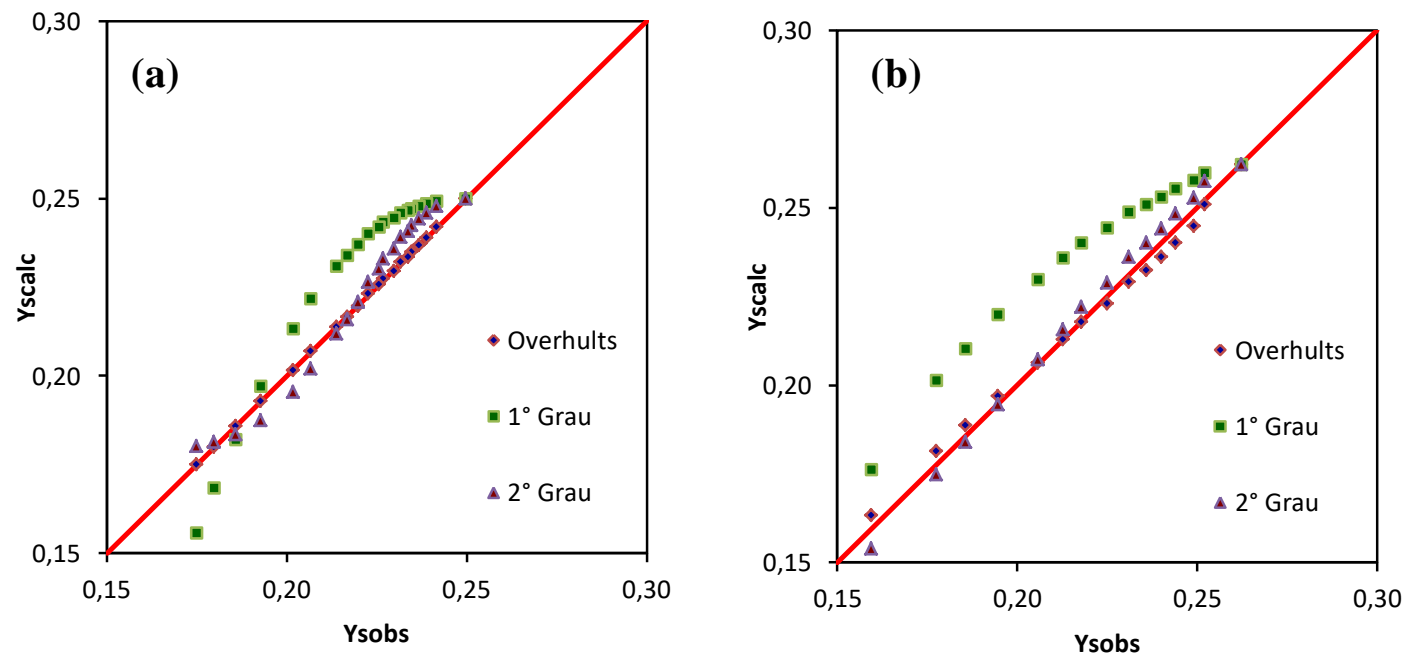

Pela análise das Figuras 2.a e 2.b, foi possível observar que os resultados obtidos pelos modelos de Overhults e de $2^{\circ}$ Grau apresentaram baixos desvios (DRM $<5 \%$ ) em relação aos valores observados, enquanto os desvios obtidos pelo modelo de $1^{\circ} \mathrm{G}$ Gau, não foram satisfatórios para que este modelo possa ser usado na simulação do processo, confirmando o que foi dito acima. 


\section{CONCLUSÕES}

O software EMSO mostrou-se uma ferramenta de fácil utilização para a simulação e a estimativa de parâmetros na secagem de grãos em camada delgada.

Com base nos dados obtidos nas simulações, foi possível verificar que o modelo de Overhults apresentou capacidade superior aos modelos de $1^{\underline{0}}$ e $2^{\underline{0}}$ Graus para prever a umidade do milho durante a secagem em camada delgada, com os menores desvios relativos médios e maiores coeficientes de determinação, podendo ser considerado o modelo mais adequado para o processo.

O modelo de taxa de secagem de $2^{\underline{0}}$ Grau, também apresentou resultados satisfatórios, considerada a incerteza de $5 \%$ nos resultados experimentais, podendo também ser aplicável, tornando-o promissor para os casos de secagem em camada espessa, uma vez que representa a própria equação de balanço de massa de água no material.

\section{AGRADECIMENTOS}

O Bolsista de Iniciação Científica agradece ao CNPq pelo auxílio financeiro.

\section{REFERENCIAS}

MANDALA, I.G., ANAGNOSTARAS, E.F., OINOKOMOU, C.K. Influence of osmotic dehydration conditions on apple air-drying kinetics and their quality characteristics. $J$. of Food Eng., v.69, p. 307-316, 2005.

MANCINI, M. C.; Transferência de massa em secadores de grãos; Tese de doutorado, 142 p., COPPE-UFRJ; 1996.

OCHOA, M. R., KESSElER, A. G., PIRONE, B. N., MARQUEZ, C. A., DE MICHELIS, A., Shrinkage during convective drying of whole rose hip (Rosa rubiginosa L.) fruits. Lebensmittel-Wissenschaft und Tech., v. 35, p. 400-406, 2002.

OVERHULTS, D.G., WHITE, G.M., HAMILTON, H.E. and ROSS, I.J., Drying of Soybeans with Heated Air. Transactions of the ASAE., pp. 112-113, 1973.

SOARES, R.P.; Manual do Software EMSO, 135 p.; 2007. 